als $1 /$ des vorhandenen Kali an Stickstoff, welcher bei dem Verkohlen und Veraschen verloren geht. Maumené schlug früher schon vor, die bei der Vorkohlung entweichenden Gase auf Ammoniak mit zu verarbeiten. Havrez ${ }^{1}$ empfahl die Verwendung des Wollschweisses zur Bereitung von Blutlaugensalz.

Vohl empfahl schon $1867,{ }^{2}$ bei der Scheidung des Abfallwassers von Tuchfabriken, Wollwäschereien u. dergl., den Seifenflüssigkeiten Chlorcalcium zuzufügen und dadurch die Seifen zu zersetzen und die Fettsäuren zu scheiden; jedoch werden hierdurch die freien Fette nicht mit gefaillt.

E. Neumann hat $1878^{3}$ ein Patent genommen für das gleiche Verfahren unter steter Wiederbenutzung des einmal gebrauchten Fällungsmittels nach Zerlegung der Kalk-Seife mittelst Salzsäure.

Meine mehrfachen Versuche haben mir mit gelöschtem Kalk, wie oben beschrieben, stets den besten Erfolg gegeben, unter Umständen war ein etwas stärkerer Zusatz geboten. Umrühren bewirkte in der Regel die sofortige Fällung der Seife und vollständige Klärung der Flüssigkeit.

E. Neumann ${ }^{4}$ hat ein weiteres Patent genommen für die Scheidung mit Kalkmilch unter Zusatz von etwas Eisenvitriol oder Bittersalz, bei Vorhandensein von leimartigen Stoffen, auch von wenig Gerbsäure. Alle diese Versuche schliessen jedoch mit der sehr lohnenden Ausnutzung der Abfallwasser der Wollwäsche, welche somit unbedingt $\mathrm{zu}$ verlangen ist.

\title{
Eine neue Bürette.
}

Von M. Vogthorr, Apotheker in Jena.

Seit dem Erscheinen der Pharm. Germ. II. hat sich das pharmaceutische Interesse auch sehr lebhaft mit der Ausbildung und Vervollkommnung der maassanalytischen Apparate beschäftigt. Die erste

1) Wagner's Jahresbericht f. techn. Chemie 16. 210.

2) Dingl. polyt. J. 185. 465. Wagn. Jabresber. 1867. 705.

3) Wagn. Jahresber. 1878. 1028.

4) Wagn. Jahresber. 1881. 967 . 
Anleitung zur Anschaffung der für die maassanalytischen Arbeiten in der Apotheke nöthigen Apparate gab Mylius in der Pharm. Centralh. 1882. No. 32. Dieselbe zeichnet sich durch gründliches Studium aller einschlägigen Arbeiten aus und kann als höchst zweckentsprechend bezeichnet werden. Von den bekannten Büretten schlägt M. die Binh'sche (englische Giess-) Bürette vor und hebt als vorzügliche Eigenschaften derselben bequeme Reinigung und verhältnissmässig geringe Zerbrechlichkeit hervor; es ist aber für den Ungeübten mit einiger Mühe verbunden, sich auf das Titriren mit dieser Bürette soweit einzuübon, dass eine sichere, genaue Analyse zu Stande kommt. Daher mag es auch gekommen sein, dass sich diese Bürette sowenig Freunde erwarb und die Meisten lieber zur Mohr'schen Quetschhahn -, zur Glashahn- oder zur Gay-Lussac'schen Giessbürette griffen und diese alle trotz grösserer Zerbrechlichkeit, trotz Mühe und Plage beim Reinigen der Bink'schen Bürette vorzogen. Man suchte dann nach allerlei Verbesserungen, die meist darauf hinausliefen, für die theure Glashahnbürette eine gleich practische, aber billigere herzustellen; trotz vieler Vorschläge ist aber nichts wirklich Lebensfähiges geschaffen werden. Gegen Ende des Vorjahres z. B. versuchte Herr Apotheker Kohlmann für seine Flaschenbürette, die Vorschluss und Bürette sugleich war, Propaganda zu machen; dieselbe erwies sich als unzweckmässig für gutes Arbeiten und der Erfinder hat die Vorwürfe, die seinem Apparate von Dr. Hübner-Jena gemacht wurden, bis heute nicht entkräften können.

Dr. Hübner hat nun selbst versucht, nicht nur das Unbrauchbare als solches zu bezeichnen, sondern auch Besseres an seine Stelle zu setzen und im Verlaufe der hierauf gerichteten Versuche hat er sich voranlasst gesehen, die ganze Idee der Flaschenbürette fallen zu lassen, und ist selbst zur gewöhnlichen Ausflussbürette wieder zurückgekehrt. Für diese war früher schon der Vorschlag geschehen, man möge zur Ersparniss der theuren, leicht zerbrechlichen Glashahnbürette, die bei Chamäleon und Silbernitratlösung nur durch Giessbüretten ersetzt werden konnte, an der oberen Oeffnung einer Messpipette einen Gummischlauch mit Quetschhahn befestigen, so erhalte man eine Bürette, bei der die Flüssigkeit mit organischen Stoffen nicht in Berührung komme und trotzdem der Glashahn ersetzt sei.

Diese Bürette hat aber den Nachtheil, dass sie sich schwer reinigen lässt, weil ihr Hals oben schr eng ist. Es war also 
M. Vogthorr, Neue Bürette.
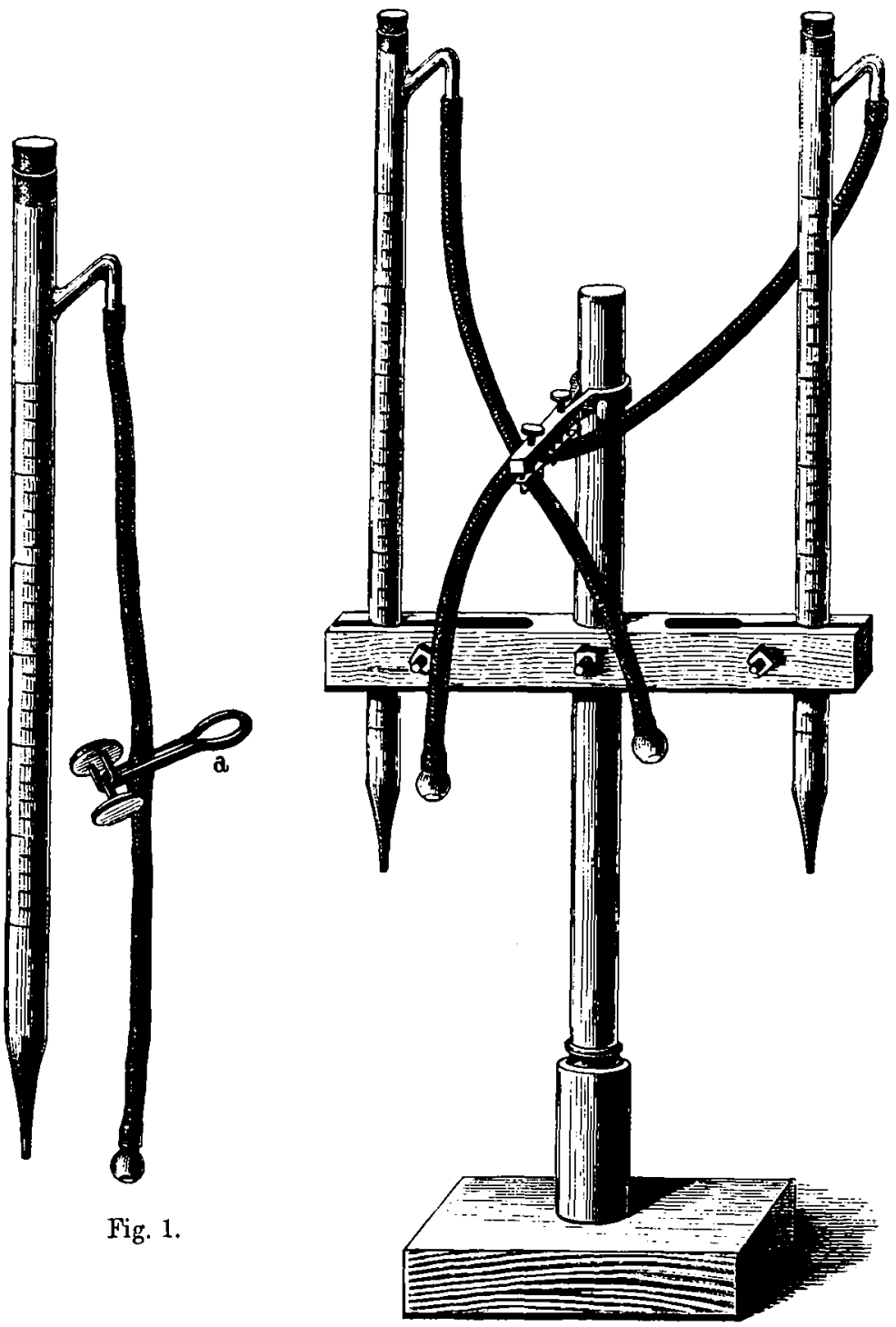

Fig. 3.

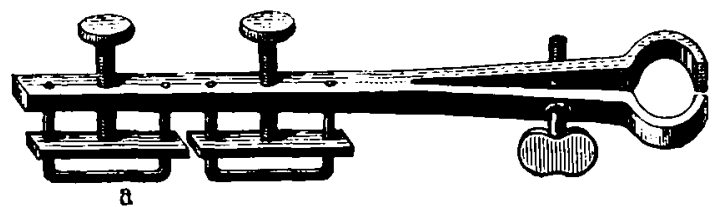

Fig. 2. 
wünschenswerth, eine neue Bürette so einzurichten, dass die Maassflüssigkeiten mit organischen Stoffen nicht in Berührung kommen, dass sich aber dennoch die Bürette leicht reinigen lässt und man die Maassflüssigkeit mit der zu genauen Arbeiten nöthigen Leichtigkeit ausfliessen lassen und zurückhalten kann. Daraus entstand nun allmählich ein Instrument von vorliegender Form (Fig. 1), welches für das deutsche Reich patentirt worden ist. Eine graduirte Röhre von 30 C.C. Inhalt ist unten in eine längere Ausflussspitze verjüngt, oben mit einem Kautschukstöpsel luftdicht verschlossen. Seitlich, in beliebiger Höhe über der Graduirung befindet sich ein kurzes, im Winkel gebogenes, engeres Glasrohr, mit schief aufsteigendem Schenkel, an dem ein Gummischlauch von beliebiger Länge, mit Mundstück versehen, befestigt ist. (Das Rolur) der Kautschukschlauch wird mit einem Quetschhahn vorsehen. Zur Füllung saugt man die Maassflüssigkeit vorsichtig in die Bürette bis über den Nullpunkt, schliesst den Quetschhahn und stellt die Flüssigkeit schliesslich durch langsames Ausfliessenlassen auf den Nullpunlit ein. Beim Oeffnen des Quetschhahnes lässt sich das Ausströmen der Flüssigkeit sehr gut reguliren, so wie auch plötzlich verhindern. Man findet sogar, dass, wenn man unmittelbar vor der Endreaction zu stehen glaubt, man den Quetschhahn gar nicht zu öffnen braucht, um noch einige Tropfen ausfliessen zu lassen; man hat nur nöthig, oberhalb des Quetschhahnes auf den Gummischlauch zu drücken, worauf noch einige Tropfen fallen werdon. Freilich kann man dann nicht verhindern, dass Luftblasen in der Flüssigkeit aufsteigen, was bei manchen Substanzen vermieden werden muss; in solchen Fällen muss dieses Verfahren dann natürlich unterbleiben. Einen Nachtheil besitzt das Instrument: Gefüllt kann es nicht tagelang hingestellt werden; denn da das über der Flüssigkeitssäule befindliche Luftvolumen allseitig abgeschlossen ist, so wird es bei der Ausdehnung durch Erwärmung einige Tropfen Flüssigkeit herausstossen; andrerseits würden beim Abkühlen einige Luftblasen durch die Flüssigkeit aufsteigen. Da dieses Stehenlassen der gefüllten Bürette leicht vermieden werden kann und durchaus nicht nothwendig ist, so fällt diese Unannehmlichkeit kaum ins Gewicht.

Aus dem Gesagten geht hervor, dass die neue Bürette den alten in Nichts nachsteht; sie hat aber den Vorzug der Billigkeit vor der Glashahnbürette (sie ist von Schlag \& Behrend in Berlin zu beziehen); auch ist sic nicht so zerbrechlich, als jene, lässt sich 
leicht reinigen und wird nicht unbrauchbar, wie jene, durch Einkitten des Glashahns bei mangelhafter Reinigung.

Wenn man nun dem Quetschhahn noch besondere Aufmerksamkeit schenken will, so wird man bald weitere Vortheile des vorliegenden Apparats entdecken.

Hübner selbst macht darauf aufmerksam, dass, wenn man statt des Mohr'schen Quetschhahnes (Fig. 1a.) einen Hoffmann'schen Schraubhahn (Fig. 2a.) verwendet, so kann man den Ausfluss des Büretten-Inhaltes für einige Zeit reguliren und bekommt dann die rechte Hand frei; da man aber zur Stellung des Quetschhahnes selbst beide Hände nöthig hat, so schlägt Hübner vor, man möge diesen Hofmann'schen Hahn in eine Klemme, vielleicht am Bürettenstativ selbst befestigen.

Dieser Vorschlag ist von wesentlicher Bedeutung. Denn indem der Quetschhahn jetzt selbst festsitzt, braucht man zur Regulirung desselben nur eine Hand und kann die andere für das untergestellte Becherglas oder dergl. verwenden. Man bekommt also eine Hand stets frei; es ist aber eine Unannehmlichkeit dabei, nämlich die, dass der Hoffmann'sche Schraubhahn sich sehr schlecht einspannen lässt und jede Klemme nur einen Quetschhahn aufnehmen kann. Dies führte mich selbst auf den Gedanken, den Quetschhahn durch Verlängerung des oberen Querbalkens mit einer Klemme zu verbinden, und da man zu einer Maassflüssigkeit meist eine Gegenflüssigkeit verwendet, so vereinigte ich zwei solcher Quetschhähne, für zwei Büretten, an einem Balken. (Fig. 2.)

Dieser kleine Apparat, der etwas mehr kostet als 2 Quetschhähne, empfiehlt sich für die Hübner'sche Pipettbürette ganz ausgezeichnet und lässt die guten Eigenschaften derselben im besten Lichte erscheinen. Einer Erklärung der Anwendung meines Quetschhahnes bedarf es nicht; ich verweise zur Erläuterung auf die beistehenden Figuren:

Figur 1. Hübner'sche Pipett-Bürette mit Mohr'schen Quetschhahn.

Figur 2. Vogtherr's Doppolquetschhahn mit Zwinge.

Figur 3. Zwei Hübnor'sche Pipett-Büretten mit Vogtherr's Doppelquetschhahn. 